\title{
Interaction between hsp90 and soluble guanylyl cyclase: physiological significance and mapping of the domains mediating binding
}

\author{
Zongmin Zhou*1, Christina Gerassimou ${ }^{1}$, Richard C Venema ${ }^{2}$, \\ Charis Roussos ${ }^{1}$, William C Sessa ${ }^{3}$, John Catravas ${ }^{2}$ and \\ Andreas Papapetropoulos ${ }^{1,4}$
}

\author{
Email: Zongmin Zhou* - apapapet@upatras.gr \\ * Corresponding author \\ from 2nd International Conference of cGMP Generators, Effectors and Therapeutic Implications \\ Potsdam, Germany, 10-12 June, 2005 \\ Published: 16 June 2005 \\ BMC Pharmacology 2005, 5(Suppl I):P65 doi:I0.1 I86/I47I-2210-5-SI-P65
}

Address: ${ }^{1}$ G.P. Livanos and M. Simou Laboratories, Evangelismos Hospital, Department of Critical Care and Pulmonary Services, University of Athens School of Medicine, Greece, ${ }^{2}$ Vascular Biology Center, Medical College of Georgia, Augusta, Georgia, USA, ${ }^{3}$ Department of Pharmacology and Program in Vascular Cell Signaling and Therapeutics, Boyer Center for Molecular Medicine, Yale University School of Medicine, New Haven, Connecticut, USA and ${ }^{4}$ Laboratory of Molecular Pharmacology, Department of Pharmacy, University of Patras, Greece

Heat shock protein 90 (hsp90) regulates stability and function of many client proteins including members of the NO-cGMP signaling pathway. Soluble guanylyl cyclase (sGC), which is the main intracellular receptor of $\mathrm{NO}$, was recently reported to be an hsp90 interacting partner. In the present study, we show that hsp90 binds to both subunits of the most common sGC form, $\alpha_{1} \beta_{1}$. Characterization of the region of hsp90 required to bind each subunit in immunoprecipitation experiments, revealed that residues 310-456 of hsp90 interact with both $\alpha_{1}$ and $\beta_{1}$. The region of $\beta_{1}$ responsible for binding to hsp90 $\beta$ was mapped using in vitro binding assays and immunoprecipitation experiments and found to lie in the regulatory domain. The physiological importance of the hsp90/sGC interaction was investigated by treating rat smooth muscle cells (RASMC) with the hsp90 inhibitors radicicol (RAD) and geldanamycin (GA) and determining both sGC activity and protein levels. Long-term ( 24 or $48 \mathrm{hr}$ ) inhibition of hsp90 resulted in a strong decrease of both $\alpha_{1}$ and $\beta_{1}$ protein levels, as well as sGC activity. Moreover, incubation of smooth muscle cells with the proteasome inhibitor MG132 blocked the GA-induced downregulation of sGC. We conclude that the $\alpha_{1}$ and $\beta_{1}$ sGC interact with the M domain of hsp90 and that this interaction regulates the pool of active sGC by affecting the protein levels of the two subunits. 